\title{
Video Article \\ Live-cell Video Microscopy of Fungal Pathogen Phagocytosis
}

\author{
Leanne E. Lewis ${ }^{1}$, Judith M. Bain ${ }^{1}$, Blessing Okai ${ }^{1}$, Neil A.R. Gow ${ }^{2}$, Lars Peter Erwig ${ }^{1,2}$ \\ ${ }^{1}$ Division of Applied Medicine, University of Aberdeen \\ ${ }^{2}$ Aberdeen Fungal Group, University of Aberdeen
}

Correspondence to: Lars Peter Erwig at I.p.erwig@abdn.ac.uk

URL: https://www.jove.com/video/50196

DOI: doi:10.3791/50196

Keywords: Infection, Issue 71, Immunology, Microbiology, Medicine, Cellular Biology, Molecular Biology, Infectious Diseases, Mycoses, Candidiasis, Bacterial Infections and Mycoses, Immune System Diseases, Live-cell imaging, phagocytosis, Candida albicans, host-pathogen interaction, pathogen, pathogen-associated molecular patterns, pattern recognition receptors, macrophage, fungus

Date Published: 1/9/2013

Citation: Lewis, L.E., Bain, J.M., Okai, B., Gow, N.A., Erwig, L.P. Live-cell Video Microscopy of Fungal Pathogen Phagocytosis. J. Vis. Exp. (71), e50196, doi:10.3791/50196 (2013).

\section{Abstract}

Phagocytic clearance of fungal pathogens, and microorganisms more generally, may be considered to consist of four distinct stages: (i) migration of phagocytes to the site where pathogens are located; (ii) recognition of pathogen-associated molecular patterns (PAMPs) through pattern recognition receptors (PRRs); (iii) engulfment of microorganisms bound to the phagocyte cell membrane, and (iv) processing of engulfed cells within maturing phagosomes and digestion of the ingested particle. Studies that assess phagocytosis in its entirety are informative ${ }^{1,2,3,4,5}$ but are limited in that they do not normally break the process down into migration, engulfment and phagosome maturation, which may be affected differentially. Furthermore, such studies assess uptake as a single event, rather than as a continuous dynamic process. We have recently developed advanced live-cell imaging technologies, and have combined these with genetic functional analysis of both pathogen and host cells to create a cross-disciplinary platform for the analysis of innate immune cell function and fungal pathogenesis. These studies have revealed novel aspects of phagocytosis that could only be observed using systematic temporal analysis of the molecular and cellular interactions between human phagocytes and fungal pathogens and infectious microorganisms more generally. For example, we have begun to define the following: (a) the components of the cell surface required for each stage of the process of recognition, engulfment and killing of fungal cells ${ }^{1,6,7,8} ;$ (b) how surface geometry influences the efficiency of macrophage uptake and killing of yeast and hyphal cells ${ }^{7}$; and (c) how engulfment leads to alteration of the cell cycle and behavior of macrophages ${ }^{9,10}$.

In contrast to single time point snapshots, live-cell video microscopy enables a wide variety of host cells and pathogens to be studied as continuous sequences over lengthy time periods, providing spatial and temporal information on a broad range of dynamic processes, including cell migration, replication and vesicular trafficking. Here we describe in detail how to prepare host and fungal cells, and to conduct the video microscopy experiments. These methods can provide a user-guide for future studies with other phagocytes and microorganisms.

\section{Video Link}

The video component of this article can be found at https://www.jove.com/video/50196/

\section{Protocol}

\section{C. albicans Growth and Conditions}

1. Prepare SC-Ura agar plates by adding $6.9 \mathrm{~g}$ yeast nitrogen base without amino acids, $1 \mathrm{ml} 1 \mathrm{M} \mathrm{NaOH}, 10 \mathrm{ml} 1 \%$ (w/v) adenine hemisulphate salt and $20 \mathrm{~g}$ technical agar (previously described in detail in ${ }^{11}$ ). Make up volume to $900 \mathrm{ml}$ with distilled $\mathrm{H}_{2} \mathrm{O}$. Autoclave, allow agar to cool, but not enough to solidify, and then add $50 \mathrm{ml}$ sterile $40 \%$ D-glucose and $50 \mathrm{ml}$ sterile $4 \%$ SC-Ura dropout under aseptic conditions. Mix, pour into Petri dishes and leave agar plates to cool and solidify. Store agar plates at $5{ }^{\circ} \mathrm{C}$ until use.

2. Streak C. albicans serotype A strain CAI4+CIp 10 from glycerol stocks stored at $-80^{\circ} \mathrm{C}$ onto SC-Ura agar plate.

3. Incubate plate at $30^{\circ} \mathrm{C}$ until colonies form and store at $5^{\circ} \mathrm{C}$.

4. Culture a single $\mathrm{C}$. albicans colony in $5 \mathrm{ml} \mathrm{SC}$-Ura medium (recipe as in 1.1 , but without technical agar) and incubate overnight at $30{ }^{\circ} \mathrm{C}$, 200 rpm to generate stationary phase $C$. albicans.

\section{C. albicans Staining Using Fluorescein Isothiocyanate (FITC)}

1. Add $10 \mu \mathrm{l} \mathrm{C.} \mathrm{albicans} \mathrm{overnight} \mathrm{culture} \mathrm{to} 990 \mu \mathrm{lPBS}(\mathrm{pH} 7.4)$ and perform a cell count using a haemocytometer.

2. To aid visualization of $C$. albicans during phagocytosis assays, stain $1 \times 10^{8} \mathrm{C}$. albicans using $1 \mathrm{mg} / \mathrm{ml} \mathrm{FITC} \mathrm{in} 0.05 \mathrm{M}$ carbonate-bicarbonate buffer $(\mathrm{pH} 9.6)$ for $10 \mathrm{~min}$ at room temperature in the dark.

3. To remove unbound FITC, wash $C$. albicans in $1 \mathrm{ml} 1 \times$ PBS, centrifuge at 3,000 $\times \mathrm{g}$ for $5 \mathrm{~min}$, remove supernatant and resuspend pellet in 1 $\mathrm{ml} 1$ x PBS. Repeat 3 times. 
4. Resuspend pellet at $1 \times 10^{6} \mathrm{cells} / \mu \mathrm{l}$ in $1 \times \mathrm{PBS}$.

\section{Preparation of the J774.1 Mouse Macrophage Cell Line}

1. Maintain $\mathrm{J} 774.1$ macrophages in $75 \mathrm{~cm}^{2}$ tissue culture flasks in DMEM medium supplemented with $10 \%$ (v/v) fetal calf serum (FCS), $200 \mathrm{U} /$ $\mathrm{ml}$ penicillin/streptomycin and $2 \mathrm{mM} \mathrm{L}$-glutamine at $37^{\circ} \mathrm{C}$ with $5 \% \mathrm{CO}_{2}$. The preparation of primary macrophages is described elsewhere in detail $^{12,13}$.

2. Scrape J774.1 cells from the tissue culture flask and transfer to a $50 \mathrm{ml}$ Falcon tube. Centrifuge at $600 \times \mathrm{g}$ for $5 \mathrm{~min}$ to obtain a cell pellet.

3. Remove supernatant and resuspend pellet in $10 \mathrm{ml}$ pre-warmed supplemented DMEM medium. Count cells using a haemocytometer and plate $1 \times 10^{6} \mathrm{~J} 774.1$ macrophages in $2 \mathrm{ml}$ supplemented DMEM medium in a $35 \mathrm{~mm}$ glass-based imaging dish. Incubate overnight at $37^{\circ} \mathrm{C}$, $5 \% \mathrm{CO}_{2}$

4. Prior to imaging, replace supplemented DMEM medium with $2 \mathrm{ml}$ pre-warmed supplemented $\mathrm{CO}_{2}$-independent medium (with $10 \%$ ( $\mathrm{v} / \mathrm{v}$ ) fetal calf serum (FCS), $200 \mathrm{U} / \mathrm{ml}$ penicillin/streptomycin and $2 \mathrm{mM} \mathrm{L-glutamine)} \mathrm{containing} 1 \mu \mathrm{M}$ LysoTracker Red DND-99.

\section{Live Cell Video Microscopy Phagocytosis Assay}

1. The choice of microscope will depend upon what is available locally, but the microscope setup will need to include an inverted stage, an environmental chamber heated to $37^{\circ} \mathrm{C}$ and excitation/emission filters for the chosen stains (FITC and TRITC).

2. Turn on the microscope heater prior to the experiment and allow sufficient time for the environmental control chamber to warm to $37^{\circ} \mathrm{C}$. The time taken for chamber temperature to stabilize will vary for different microscope setups.

3. Turn on the microscope and computer, and load the imaging software. Mount the imaging dish on the microscope stage and adjust the focus to find the J774.1 macrophages. Optimize the appearance of TRITC and DIC images by adjusting the percentage of transmitted light and exposure times.

4. Remove the imaging dish and add $3 \times 10^{6}$ FITC-stained C. albicans to the dish. Record the time that $C$. albicans is added to the dish. Return the dish to the stage and optimize the appearance of FITC images if necessary. Set up a points list if required.

5. Commence imaging when all points are in focus and the channels are optimized. Capture FITC, TRITC and DIC images every minute for 6 hr.

\section{Representative Results}

Here we show representative results for $C$. albicans uptake by a murine macrophage cell line $\mathrm{J} 774.1$ during a 6 hr live-cell video microscopy experiment. Figure 1 is a representative live-cell video microscopy movie, showing phagocytosis of $C$. albicans by murine J774.1 macrophages. Live-cell video microscopy enables internalization of $C$. albicans to be visualized without the need to stain external Candida. However, during these experiments, $\mathrm{C}$. albicans was stained using the $\mathrm{pH}$-sensitive dye FITC (green), which is quenched during acidification of the macrophage phagosome, and facilitates confirmation that Candida has been internalized (Figure 1). To further aid visualization of ingested $C$. albicans, macrophages were stained using the red fluorescent dye LysoTracker red DND-99, which stains acidic compartments and serves as a nonspecific marker of phagosome maturation. Figure $\mathbf{2}$ is a still image from a live-cell video microscopy experiment and illustrates the variation in the number of $C$. albicans ingested by individual J774.1 macrophages. In this experiment, $82 \%$ of $J 774.1$ macrophages engulfed at least one C. albicans cell at the end of the $6 \mathrm{hr}$ phagocytosis assay. In Figure 3, the number of internalized C. albicans cells per macrophage is reported. The mean number of $C$. albicans taken up per macrophage is 3.4, but interestingly macrophages can ingest up to 16 fungal cells. Figure 4 is a sequence of still images from a representative live-cell video microscopy experiment showing a macrophage phagocytosing $C$. albicans cells, hypha growth with the macrophage and ultimately macrophage lysis. Figure 4 illustrates that video microscopy enables analysis of events following engulfment of target cells, i.e. data can be generated for the killing of macrophages by $C$. albicans hyphae in relation to the number and morphogenesis of ingested target cells, and how macrophage killing relates to phagosome maturation which can be studied in real time.

Figure 1. Live-cell video microscopy movie showing phagocytosis of $C$. albicans by murine $J 774.1$ macrophages. Macrophages are stained using the red fluorescent dye LysoTracker red DND-99, and C. albicans are stained using FITC (green). Macrophages and C. albicans were cocultured for a period of $6 \mathrm{hr}$ at $37^{\circ} \mathrm{C}$ in complete $\mathrm{CO}_{2}$-independent medium. Images were captured at 1 min intervals for 6 hr. Macrophages can be seen establishing cell-cell contact with $C$. albicans, which is then followed by uptake of $C$. albicans into macrophage phagosomes. Arrows point to $C$. albicans prior to engulfment by J774.1 macrophages. This video also shows that FITC fluorescence is quenched following engulfment of $C$. albicans. Scale bar, $10 \mu \mathrm{m}$. Click here to view movie. 


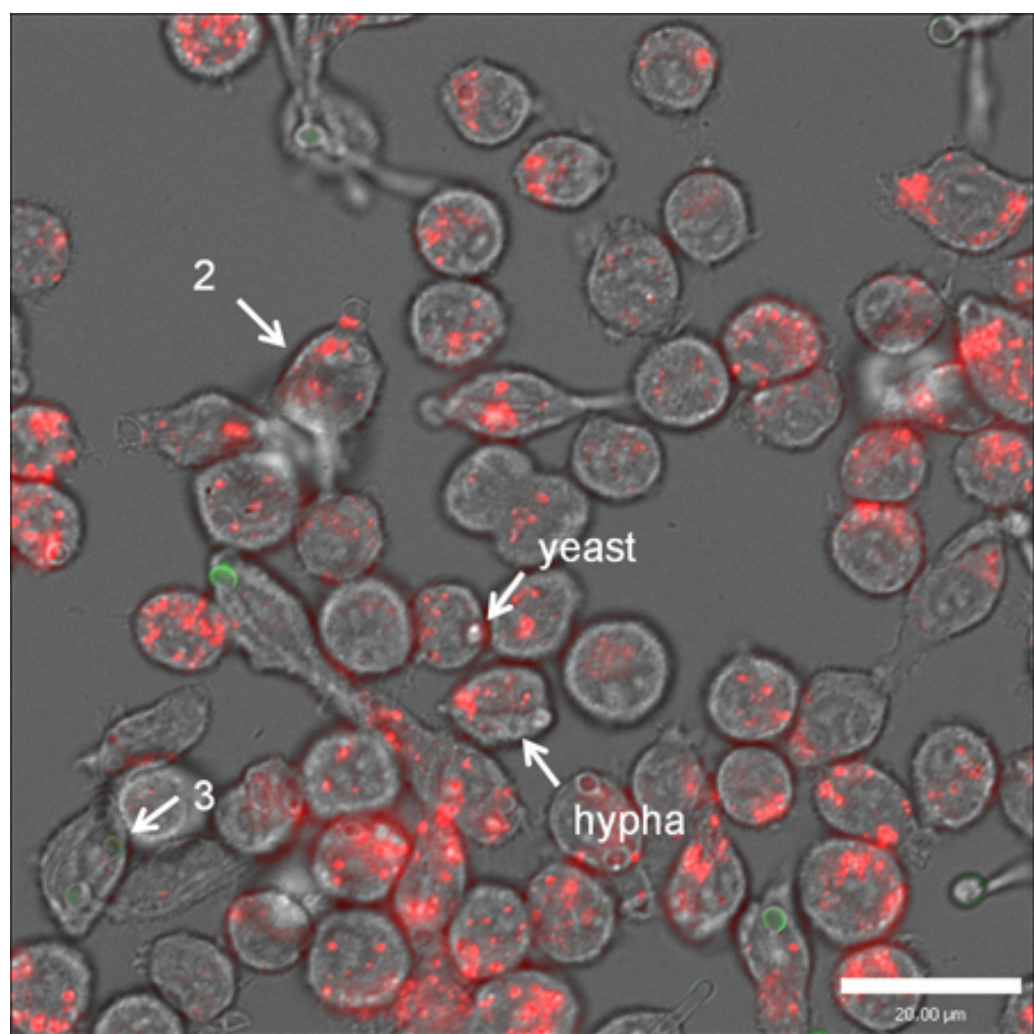

Figure 2. Representative live-cell video microscopy still image showing variation in the number of C.albicans engulfed by individual macrophages. Macrophages (stained using LysoTracker red DND-99) were co-cultured with FITC-stained C. albicans (green). There is variation in the number of $C$. albicans engulfed (a number next to an arrow indicates the number of $C$. albicans engulfed), and in $C$. albicans morphology (i.e. yeast verses hypha). Scale bar, $20 \mu \mathrm{m}$.

\section{The number of $C$. albicans ingested by individual $\mathrm{J} 774.1$}

\section{macrophages}

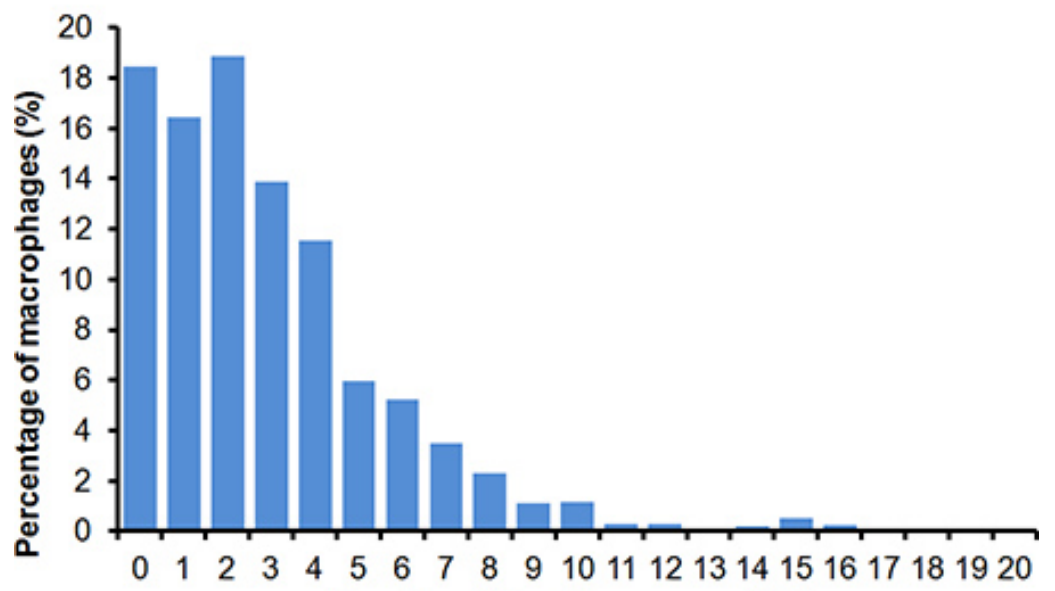

Number of $C$. albicans engulfed

Figure 3. Graph showing the number of $C$. albicans ingested per J774.1 murine macrophage at the end of 6 hr phagocytosis assays. The data represent 2 independent experiments. A total of 100 macrophages were counted from 3 fields from each experiment, giving a total of 600 individual macrophages. 

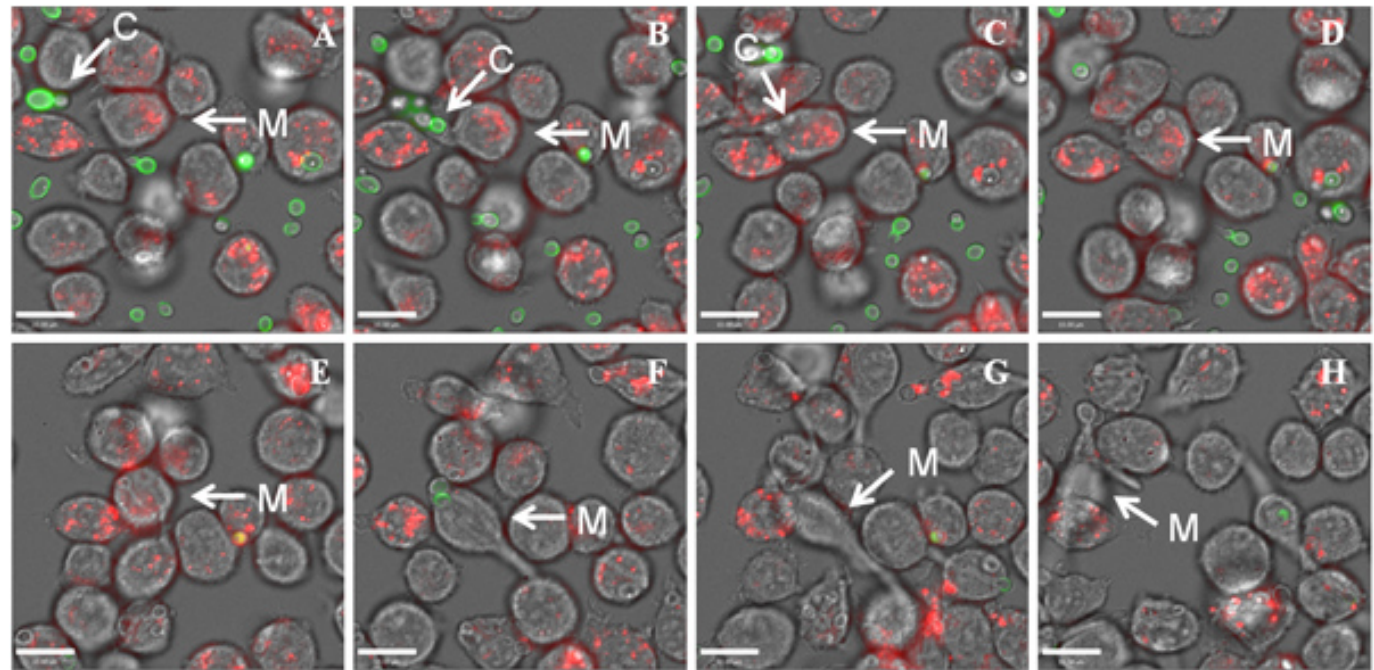

Figure 4. A series of images from a representative live-cell video microscopy experiment showing a macrophage (M) and $C$. albicans (C) prior to and during recognition (A, B), and during and after uptake (C, D). C. albicans hyphae continue to grow within the macrophage (E, F), which can result in macrophage lysis $(G)$. Other macrophages are recruited to the site of rupture and attempt to ingest the released $C$. albicans $(\mathrm{H})$. Macrophages are stained using the red fluorescent dye LysoTracker red DND-99, and C. albicans are stained using FITC (green). Note that FITC staining is quenched following C. albicans uptake by J774.1 macrophages (C). Scale bar, $10 \mu \mathrm{m}$.

Discussion

Here the method for the use of live-cell video microscopy to study macrophage phagocytosis is described. Video microscopy offers multiple additional layers of information for analysis. One basic advantage is that uptake data can be generated (from a single experiment) for any time point throughout the $6 \mathrm{hr}$ observation period. More importantly, the method described enables differential analysis of the individual stages of phagocytosis. We have, for example, shown that changes in overall uptake of $C$. albicans glycosylation and morphogenesis mutants by macrophage cell lines and primary macrophages can be either a consequence of changes in macrophage migration towards target cells or rate of engulfment once cell-cell contact is established ${ }^{7}$.

There are a number of pitfalls to avoid when conducting these experiments. First, it is very important to ensure stable environmental conditions throughout the experimental procedure. This is best achieved in an environmental chamber that is set to the experimental conditions several hours before commencing the experiment. Video quality is highly dependent on sophisticated modules that use infrared lasers to automatically maintain the sample z-position regardless of mechanical or thermal changes thus eliminating the need for manual focus corrections.

Given the extended nature of the experiments, it is important to limit laser light exposure and associated photobleaching and photoconversion effects, which can be minimized by employing highly fluorescent markers, which facilitate low exposure times. The FITC staining protocol described here is quick and reliable. As FITC is a very bright and stable stain, low exposure times are required and this makes FITC ideal for extended time-lapse movies. However, we routinely use a variety of different target stains, including Calcofluor White and PKH dyes as well as tagged organisms.

Most of our published work is conducted using a wide-field microscope, but exposure can be further minimized by using a spinning disc confocal microscope and in our hands this is essential for time-lapse 3D video microscopy.

During this study, images were captured at 1 min intervals over a $6 \mathrm{hr}$ phagocytosis assay. The interval between images can be adjusted depending on the process under investigation. For example, the interval can be decreased when investigating rapid processes such as vesicular trafficking. The minimum time interval will be limited by microscope and camera specifications. There are some considerations to bear in mind when adjusting timings. First, decreasing the interval between snapshots will mean fewer points can be imaged and file size will be considerably increased. On the contrary, increasing the image interval too much will make the movie lose continuity.

This approach can be applied in principle to study other pathogens and uptake of dying host cells. For example, we have recently shown that bone-marrow derived macrophages from sialoadhesin-deficient mice exhibit greatly reduced binding and phagocytosis of sialylated Campylobacter jejuni ${ }^{8}$. However, target size is an important determinant for feasibility, as image analysis becomes increasingly difficult with a reduction in target cell size. Sophisticated image analysis software is essential for rapid video microscopy analysis, and this should be coupled with appropriate bioinformatics support to facilitate the generation of algorithms to study migration of individual cells and entire cell populations 7 . Live-cell video microscopy in combination with sophisticated image analysis software for the minute-by-minute analysis of migration and individual macrophage-C.albicans interactions, provides unique insight into the complexity of $C$. albicans phagocytosis by macrophages. There is enormous potential to expand these methods to study other pathogens and phagocytes (dendritic cells, neutrophils) and to develop 3D video microscopy to image cell-cell interactions in greater detail or on more physiological surfaces such as epithelial and endothelial cell layers. This technique is part of the next generation of tools in the study of host-pathogen interactions and will help generate detailed spatial and temporal information on a broad range of dynamic processes. 


\section{Disclosures}

The authors have nothing to disclose. NG is a recipient of unrestricted grant support for basic research by Gilead Sciences. LE works as a consultant for GSK in early drug development.

\section{Acknowledgements}

LPE is a Scottish Senior Clinical Fellow and acknowledges the support of the Chief Scientist Office (SCD/03). This work was funded by Wellcome Trust Project Grant to LPE (089930). NARG was funded by a Wellcome Trust Programme Grant (080088) and an equipment Grant (075470) (for DeltaVision), and by a FP7-2007-2013 Grant (HEALTH-F2-2010-260338-ALLFUN). We would like to thank the University of Aberdeen imaging facility, in particular Kevin MacKenzie, for helpful support and advice.

\section{References}

1. McKenzie, C.G.J., et al. Contribution of Candida albicans cell wall components to recognition by and escape from murine macrophages. Infect. Immun. 78, 1650-1658 (2010).

2. Mora-Montes, H.M., et al. Recognition and blocking of innate immunity cells by Candida albicans chitin. Infect. Immun. 79, 1961-1970 (2011).

3. Keppler-Ross, S., et al. Recognition of yeast by murine macrophages requires mannan but not glucan. Eukaryot. Cell. 9, 1776-1787 (2010).

4. Vijayan, D., et al. Mincle polarizes human monocyte and neutrophil responses to Candida albicans. Immunol. Cell Biol. doi:10.1038/ icb.2012.24 (2012).

5. Seider K., et al. The facultative intracellular pathogen Candida glabrata subverts macrophage cytokine production and phagolysosome maturation. J. Immunol. 187, 3072-3086 (2011).

6. Sheth C., et al. Glycosylation status of the $C$. albicans cell wall affects the efficiency of neutrophil phagocytosis and killing but not cytokine signalling. Med. Mycol. (5), 513-524 (2011).

7. Lewis, L.E., et al. Stage specific assessment of Candida albicans phagocytosis by macrophages identifies cell wall composition and morphogenesis as key determinants. PLoS Pathog. 8 (3), e1002578 (2012).

8. Klaas, M., et al. Sialoadhesin Promotes Rapid Proinflammatory and Type I Interferon Responses to a Sialylated Pathogen, Campylobacter jejuni. J. Immunol., In Press, (2012).

9. Lewis, L.E., et al. Candida albicans infection inhibits macrophage cell division and proliferation. Fungal Genet. Biol., (2012).

10. Bain, J.M., et al. Non-lytic expulsion/exocytosis of Candida albicans from macrophages. Fungal Genet. Biol., (2012).

11. Mora-Montes, H., et al. Interactions between macrophages and cell wall oligosaccharides of Candida albicans. Methods Mol. Biol. 845, 247-60 (2012).

12. McPhillips, K., et al. Assessment of apoptotic cell phagocytosis by macrophages. Methods Mol. Biol. 559, 247-256 (2009).

13. Erwig, L-P., et al. Differential regulation of phagosome maturation in macrophages and dendritic cells mediated by Rho GTPases and ezrinradixin-moesin (ERM) proteins. Proc. Natl. Acad. Sci. U.S.A. 103 (34), 12825-30 (2006). 